\title{
Prevalence of chronic kidney disease in the Lazio region, Italy: a classification algorithm based on health information systems
}

\author{
Claudia Marino ${ }^{1^{*}}$, Pietro Manuel Ferraro ${ }^{2,3}$, Matteo Bargagli ${ }^{2,3}$, Silvia Cascini ${ }^{1}$, Nera Agabiti ${ }^{1}$, \\ Giovanni Gambaro ${ }^{4}$ and Marina Davoli ${ }^{1}$
}

\begin{abstract}
Background: Estimating CKD prevalence is difficult. Information on CKD prevalence is rather scanty in Italy and available figures come from surveys in selected geographical areas. Administrative data have been already demonstrated to be an effective tool in estimating the epidemiological burden of diseases, however there is limited experience in literature as far as CKD is concerned.

Methods: The aim of this study is to develop an algorithm based on regional Health Administrative Databases to identify individuals with CKD and provide estimates of disease prevalence in Lazio Region (Italy); about 5.500.000 inhabitants in 2017. A population-level analysis based on a record-linkage strategy using data from Health Administrative Databases has been applied in Lazio Region. CKD cases were identified between January 1, 2012 and December 31, 2017 using Outpatient Specialist Service Information System, Hospital Discharge Registry, Ticket Exemption Registry and Drug Dispensing Registry. Age-specific and standardized prevalence rates were calculated by gender. CKD cases were classified as higher and lower severity.

Results: The algorithm identified 99,457 individuals with CKD (mean age 71 years, 55.8\% males). The exclusive contributions of each regional source used were: 35,047 (35.2\%) from Outpatient Specialist Service Information System, 27,778 (27.9\%) from Hospital Discharge Registry, 4143 (4.2\%) from Ticket Exemption Registry and 463 (0.5\%) from Drug Dispensing Registry; $5.1 \%$ of cases were found in all databases. The standardized prevalence rate at December 31, 2017 was 1.76, 2.06\% for males and 1.50\% for females. The prevalence increased with age, rising from $0.33 \%$ (age $0-18$ ) up to $14.18 \%$ (age $85+$ ) among males and from $0.25 \%$ up to $8.18 \%$ among females. The proportion of CKD individuals with lower severity disease was $78.7 \%$ in both genders.

Conclusions: The proposed algorithm represents a novel tool to monitor the burden of CKD disease, that can be used by the regional government to guide the development and implementation of evidence-based pathways of care for CKD patients. The high prevalence of people with CKD of lower severity should be carefully considered in order to promote diagnosis and optimal management at early stages.
\end{abstract}

Keywords: Administrative data, Chronic kidney disease, Prevalence, Diagnostic algorithm, Health administrative datasets

\footnotetext{
* Correspondence: c.marino@deplazio.it

1 Department of Epidemiology Lazio Regional Health Service, Via Cristoforo

Colombo, 112, 00147 Roma, Italy

Full list of author information is available at the end of the article
}

(c) The Author(s). 2020 Open Access This article is distributed under the terms of the Creative Commons Attribution 4.0 International License (http://creativecommons.org/licenses/by/4.0/), which permits unrestricted use, distribution, and reproduction in any medium, provided you give appropriate credit to the original author(s) and the source, provide a link to the Creative Commons license, and indicate if changes were made. The Creative Commons Public Domain Dedication waiver (http://creativecommons.org/publicdomain/zero/1.0/) applies to the data made available in this article, unless otherwise stated. 


\section{Background}

Chronic kidney disease (CKD) is a common chronic condition, which may often lead to renal failure. It increases the risk of cardiovascular complications and is linked to a variety of signs and symptoms, evidence of multi-organ dysfunction such as chronic anemia, inflammation, mineral bone disease and sarcopenia [1]. A recent metaanalysis showed that CKD prevalence is probably underestimated and higher than that of diabetes; in fact, worldwide CKD prevalence was $13.4 \%$ for stages $1-5$ and $10.6 \%$ for stages 3-5 [2, 3]. An Italian epidemiological study with data at national level reported prevalence rates of $7.5 \%$ among males and $6.5 \%$ among females for the age category 35-79 years [4]. Therefore, CKD encompasses a variety of disorders and represents a true major public health burden [3]. The economic burden of CKD is substantial. Disease stage is predictive of higher costs, with particularly burdensome expenditures for end-stage renal disease (ESRD) due to renal replacement therapy (dialysis or transplantation) [5]. Although most of the costs per patient in the CKD population is related to ESRD, earlier stages also generate costs, mainly by inducing cardiovascular events [6]. Actually, costs in the earlier stages of CKD before dialysis are less investigated [7].

In Italy, the estimated annual cost of pre-dialysis patients is $€ 7422$ for CKD stage IV and $€ 8971$ for stage V. The costs are significantly higher for ESRD patients: the actual cost per year for dialysis patients is $€ 29,800$ for those on peritoneal dialysis and $€ 43,800$ for those on hemodialysis $[8,9]$. Indirect costs should be added. Based on these data, it has been supposed that delaying the progression from CKD stage III to IV for $10 \%$ of affected patients for a 5-year period will save up to $€ 2.5$ billion [10]. Hence, prevention, early diagnosis, management and care for people with CKD have high impact on health care programs in terms of direct and indirect costs. A working national or regional recognition program for CKD could allow to establish an early diagnosis, reducing health care costs and improving quality of life for the patients. Currently, a system with such characteristics is missing in Italy and, to the best of our knowledge, in Europe. Since it is difficult to assess CKD in large-scale models based on laboratory parameters only, there is actually increasing interest in the use of administrative data for epidemiological purposes.

Administrative data have been already demonstrated to effectively estimate prevalence of cardiovascular disease and diabetes with high sensitivity and specificity $[11,12]$. In order to achieve the same results, a systematic review analyzed 13 administrative database-coding algorithms for kidney disease, but it found low sensitivity and variable positive predictive value regarding CKD [13].

In Italy, a recent systematic review highlighted the paucity of works conducted to identify people with CKD based on routinely collected data [14]. CKD has multiple causes and clinical pictures, with increasing levels of severity. While more advanced disease can be captured in the administrative databases by intercepting the prescription of specific drugs and procedures, milder stages of disease need to be assessed through more complex, integrated multi-sources algorithms. Unfortunately, to the best of our knowledge no such approach has ever been applied.

There is a need to measure the complete burden of CKD and develop procedures based on administrative data, in order to monitor temporal and geographic variation in occurrence, to evaluate quality of care for these patients, and to support the implementation of new organizational integrated care model [15]. In Italy, regional registries on dialysis exist, and there is a coordination effort at national level to describe and monitor this stage of the disease (https://ridt.sinitaly.org/). Moreover, the Italian Ministry of Health in 2017 promoted the institution of the national CKD registry - including all stages - to describe the epidemiology, to monitor the quality of care and health outcomes, and to prevent the incidence of the most severe - and costly - stages of this disease [10].

\section{Methods}

The aim of this study is to develop an algorithm based on health information systems, to identify people with CKD and to provide prevalence estimates in Lazio, a highly-populated region in central Italy. Such approach could potentially be extended in other Italian regions.

\section{Data sources}

\section{Health administrative data}

Different health information systems were used to identify the population with CKD in the Lazio region (over 5,700, 000 residents). The Hospital Discharge Registry (HDR) routinely collects data from all regional hospitals, including information on patients' demographic characteristics, up to six discharge diagnoses and up to six hospital procedures codes according to the International Classification of Disease-Ninth Revision-Clinical Modification (ICD-9CM). HDR also collects data on inter-regional mobility, all hospitalizations of Lazio residents that occur outside the region. The Ticket Exemption Registry (TER) includes data on all residents who are entitled to co-pay fee exemption for some particular conditions, e.g. disability, chronic diseases, low income or old age. The Outpatient Specialist Service Information System (OSSIS) collects data from outpatient clinics (e.g. whether the participant underwent visits, specialized diagnostic-instrumental services and laboratory analyses). The Drug Dispensing Registry (PHARM) collects individual records for each drug prescription that is dispensed from public and private pharmacies and by hospital at discharge. Drugs are identified 
Table 1 Diagnosis (code = ICD-9-CM), procedures (code =ICD-9-CM), outpatient services (code = Regional codification) and drugs (code $=$ ATC), name and code

Name $\quad$ Code (diagnostic branch)

The Hospital Discharge Registry (HDR)

Diagnosis

Diabetes with renal manifestations $\quad 250.4 X$

$\begin{array}{ll}\text { Hypertensive chronic kidney disease } & \text { 403.XX }\end{array}$

$\begin{array}{ll}\text { Hypertensive heart and chronic kidney disease } & \text { 404.XX }\end{array}$

$\begin{array}{ll}\text { Chronic glomerulonephritis } & \text { 582.XX }\end{array}$

$\begin{array}{ll}\text { Nephritis and nephropathy not specified as acute or chronic } & \text { 583.XX }\end{array}$

$\begin{array}{ll}\text { Chronic kidney disease (ckd) } & \text { 585.XX }\end{array}$

$\begin{array}{ll}\text { Renal failure, unspecified } & \text { 586.XX }\end{array}$

$\begin{array}{ll}\text { Renal sclerosis, unspecified } & \text { 587.XX }\end{array}$

Disorders resulting from impaired renal function $\quad 588 . X X$

$\begin{array}{ll}\text { Cystic kidney disease } & 753.1 \mathrm{X}\end{array}$

$\begin{array}{ll}\text { Chronic pyelonephritis } & \text { 590.0X }\end{array}$

Encounter for dialysis and dialysis catheter care V56.X

Kidney replaced by transplant $\quad$ V42.0

Procedures

$\begin{array}{lr}\text { Hemodialysis } & 39.95\end{array}$

$\begin{array}{lr}\text { Peritoneal dialysis } & 54.98\end{array}$

$\begin{array}{ll}\text { Transplant of kidney } & 55.6 \mathrm{X}\end{array}$

$\begin{array}{lr}\text { Arteriovenostomy for renal dialysis } & 39.27\end{array}$

$\begin{array}{ll}\text { Creation of cutaneoperitoneal fistula } & 54.93\end{array}$

Revision of arteriovenous shunt for renal dialysis 39.42

$\begin{array}{ll}\text { Removal of arteriovenous shunt for renal dialysis } & 39.43\end{array}$

$\begin{array}{ll}\text { Venous catheterization for renal dialysis } & 38.95\end{array}$

Closed [percutaneous] [needle] biopsy of kidney 55.23

Complex outpatient services for

Assess diagnosis of nephropathies $\quad$ P583

Assess chronic kidney disease $\quad$ P585A

Assess kidney transplant $\quad$ P585B

$\begin{array}{lr}\text { Follow-up of kidney transplant patient } & \text { PV420 }\end{array}$

\section{Outpatient Specialist Service Information System (OSSIS)}

Services

$\begin{array}{lr}\text { First ambulatory specialist visit (nephrology) } & 89.7 \text { (29) }\end{array}$

$\begin{array}{lr}\text { Ambulatory specialist visit (nephrology) } & 89.01(29)\end{array}$

$\begin{array}{ll}\text { Measurement of urine albumin } & 90.33 .4\end{array}$

$\begin{array}{ll}\text { Definition of the haemodialysis or peritoneal dialysis scheme (nephrology) } & 89.03 \text { (29) }\end{array}$

Hemodialysis or hemodiafiltration $\quad 39.95 . X$

$\begin{array}{ll}\text { Peritoneal dialysis } & \text { 54.98.X }\end{array}$

Venous catheterization for renal dialysis 38.95

Creation of cutaneoperitoneal fistula (peritoneal catheter) 54.93

$\begin{array}{ll}\text { Debriding of peritoneal catheter } & 39.99 .1\end{array}$

Removal of peritoneal catheter $\quad 97.82$

$\begin{array}{lr}\text { Revision of peritoneal catheter } & 97.29 .1\end{array}$ 
Table 1 Diagnosis (code $=I C D-9-C M)$, procedures (code =ICD-9-CM), outpatient services (code = Regional codification) and drugs (code $=$ ATC), name and code (Continued)

\begin{tabular}{ll}
\hline Name & Code (diagnostic branch) \\
\hline Drug Dispensing Registry (PHARM) & ATC \\
Erythropoietin & B03XA01 \\
Darbepoetin alfa & B03XA02 \\
Methoxy polyethylene glycol-epoetin beta & B03XA03 \\
Polystyrene sulfonate & V03AE01 \\
Sevelamer & V03AE02 \\
Lanthanum carbonate & V03AE03 \\
Sucroferric oxyhydroxide & V03AE05 \\
\hline
\end{tabular}

by the national drug register code, which refers to the International Anatomical Therapeutic Chemical Classification System (ATC). Individual patient data and date of dispensing are reported for each prescription. The Regional Register of Causes of Death lists the causes of death, coded according to ICD-9 revision, for all deaths of citizens residing in the region. Finally, the Regional Health Assistance File contains the history of cancellation and registrations of the residence for each citizen ensured by the Regional Health Service. The Lazio Dialysis Registry (LDR) is a population-based registry that started in 1994. It collects detailed information on all patients undergoing chronic dialysis (e.g., those undergoing either hemodialysis or peritoneal dialysis for a period of at least 90 days). It supports the planning, management, control and evaluation of health care, as well as the study and scientific research activities in the medical, biomedical and epidemiological fields. All dialysis units of the Lazio region are requested by law to register the information on their patients and to update it every 6 months.

All residents of the Lazio region served by the public health service have a personal identification number recorded in all the regional healthcare databases. This individual identifier provides the key to link all regional databases and allows to identify individuals uniquely within the regional health system.

\section{Study population \\ Case definition criteria}

To identify cases with CKD at December 312,017 we used HDR, TER, OSSIS and (PHARM) during the time from January 012012 to December 312,017.

From HDR, we selected all subjects with at least one hospital discharge (primary or secondary diagnosis) or procedures referred to codes reported in Table 1. To avoid selecting acute renal failure, records of hospitalization selected only with procedures 39.95 (hemodialysis) or 38.95 (venous catheterization for renal dialysis) and in which one of the diagnosis codes was
584.XX (acute renal failure) were eliminated. From TER, we selected all subjects registered at the date of prevalence with CKD (code: 025.585) or kidney transplantation (code: 025.V42.0). From OSSIS, we selected all subjects who had at least one record with codes listed in Table 1 and we considered only subjects who had undergone at least two nephrological visits or one nephrological visit plus at least one measurement of urine albumin or at least one prescription of the drugs listed in Table 1 (data source PHARM).

All patients who satisfied at least one of the above conditions were selected. We excluded patients who died during the period or patients who did not reside in the Lazio region at December 312,017.

\section{Identification of CKD cases with different levels of severity.}

CKD cases were classified into two levels of severity. Higher severity cases were subjects who during the selection period had undergone at least one dialysis or kidney transplant or one hospitalization with diagnosis code of CKD stage IV or greater (ICD-9-CM diagnosis code: $585.4 \mathrm{X}, 585.5 \mathrm{X}, 585.6 \mathrm{X})$ or who had been prescribed at least one of the drugs listed in Table 1. Lower severity cases were considered all the remaining individuals in the prevalent population.

\section{Statistical analysis Prevalence rates}

The prevalence estimates were estimated at December 312,017, named prevalence date. Age- and gender-specific prevalence rates $(\times 100)$ were calculated by dividing the number of patients with CKD alive and resident at the prevalence date by the number of residents in the Lazio region. The age classes considered were $0-18,19-44,45-64,65-74,75-84$, $85+$. Additionally, we calculated standardized prevalence rates \% (direct method) separately by gender and severity of CKD, using the Italian ISTAT population at December 312,017 as reference population. 
For all measures reported, 95\% Confidence Intervals $(95 \% \mathrm{CI})$ were estimated.

Validity of the dialysis case ascertainment algorithm.

The availability of the Lazio Dialysis Registry data gave us the opportunity to evaluate the validity of our algorithm in the identification of a sub-group out of the whole CKD population, namely those patients who received dialysis during the selection period. Patients undergoing chronic dialysis between January 012012 and December 312,017 alive and resident at the prevalence date were selected from the Lazio Dialysis Registry and were linked with the prevalent cases who had received dialysis treatments according to the algorithm. Considering Lazio Dialysis Registry cases as the gold standard for dialysis treatments, we calculated sensitivity, specificity, positive and negative predictive values of the algorithm $[15,16]$.

All analyses were performed using SAS Version 9.4. This study was carried out in full compliance with the current privacy laws. It was based on anonymous computer records from health information systems and did not require ethical approval.

\section{Results}

The algorithm identified a population with CKD of 99, 457 individuals at December 312,017 (Fig. 1).

Males were $55.8 \%$ of the total, mean age was 70 years (standard deviation (SD) 17.7) for males and 72 years (SD 18.0) for females ( $p$-value $<0.0001$ for the difference between males and females). The exclusive contributions from each source to the identification of CKD cases were: 35,047 (35.2\%) from OSSIS, 27,778 (27.9\%) from HDR, 4143 (4.2\%) from TER and 463 (0.5\%) from

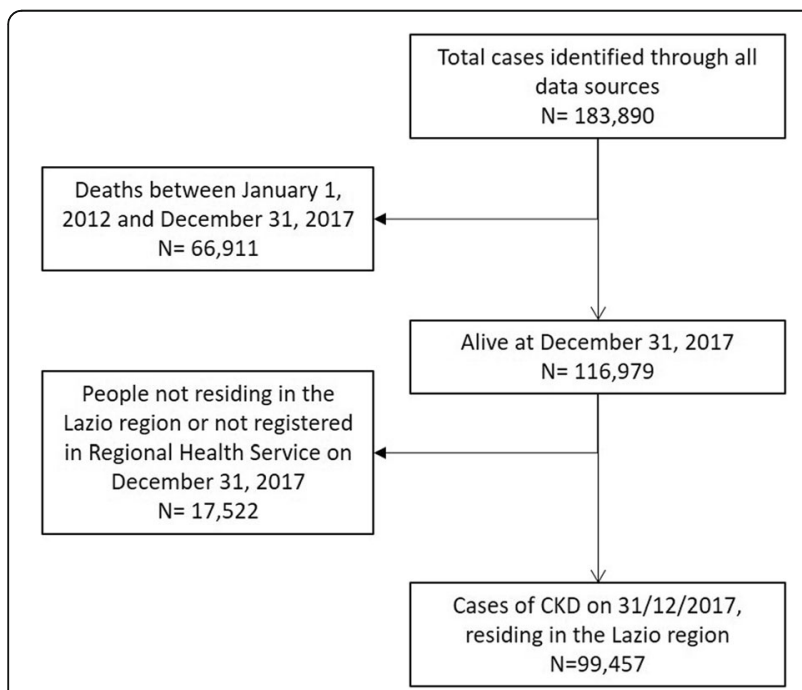

Fig. 1 Flow-chart selection of Chronic Kidney Disease (CKD) prevalence cases at December 312,017, resident in the Lazio region
PHARM; $5.1 \%$ of cases were found in all databases (Fig. 2).

The crude prevalence rate of CKD in the Lazio region was $1.76 \%$ (95\%CI $1.75,1.78), 2.06 \%$ for males and $1.50 \%$ for females. The prevalence increased by age group, from $0.33 \%$ (age $0-18$ ) up to $14.18 \%$ (age $85+$ ) in males and from $0.25 \%$ up to $8.18 \%$ in females (Table 2). The lowest male to female ratio was in age class 19-44 and the highest in age class 65-74 (1:14 and 1:83, respectively). The standardized prevalence was $2.35 \%$ (95\% CI $2.33,2.37)$ for males and $1.39 \%$ (95\% CI 1.38, 1.40) for females, with a male to female ratio of 1:69 (Table 2).

The algorithm identified 21,159 higher severity cases (21.3\% of all CKD cases). Males were $55.8 \%$ of the total, and mean age for males and females was 71 (SD 15.3) and 74 (SD 15.8) years, respectively. The exclusive contributions from each source to the identification of higher severity CKD cases were: 116 (0.5\%) from OSSIS, 2400 (11.3\%) from HDR, 219 (1.0\%) from TER and 463 (2.2\%) from PHARM; $24.0 \%$ of cases were found in all databases (Fig. 3).

For males, the standardized prevalence rate was $0.50 \%$ (95\% CI $0.50,0.51)$ for higher severity and 1.85\% (95\% CI 1.83, 1.87) for lower severity; the corresponding values for females were $0.29 \%$ (95\% CI $0.29,0.30)$ and $1.10 \%$ (95\% CI 1.09, 1.11). Looking at age-specific prevalence, no difference was observed in the age class 0-18 in higher severity by gender. The prevalence of higher severity cases increased across age classes from $0.02 \%$ up to $3.14 \%(0-18,85+$ respectively) for males and from $0.02 \%$ up to $2.11 \%$ for females (Fig. 4 ).

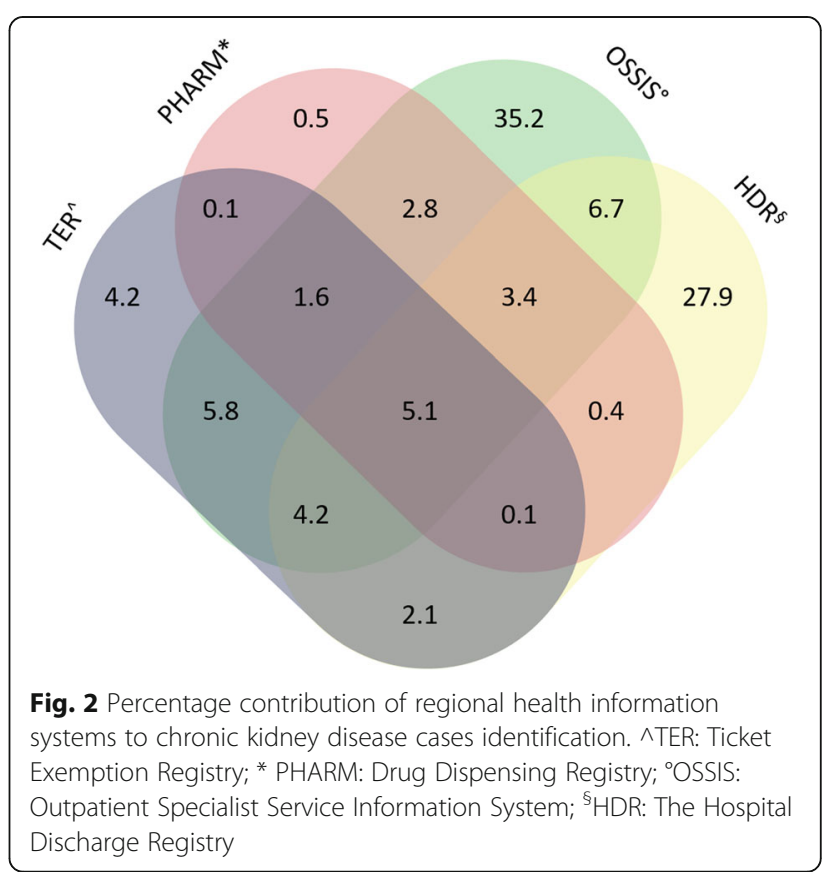


Table 2 Age- and gender-specific prevalence per 100 population on December 312,017, Lazio region, Italy

\begin{tabular}{|c|c|c|c|c|c|c|c|c|c|c|c|c|c|c|c|c|}
\hline \multirow{3}{*}{$\begin{array}{l}\text { Age } \\
\text { class }\end{array}$} & \multicolumn{5}{|l|}{ Males } & \multicolumn{5}{|c|}{ Females } & \multicolumn{5}{|l|}{ Total } & \multirow{3}{*}{$\begin{array}{l}\text { Rate } \\
\text { M/F }\end{array}$} \\
\hline & \multirow[t]{2}{*}{ Cases } & \multirow[t]{2}{*}{ Pop $^{a}$} & \multirow{2}{*}{$\begin{array}{l}\text { Prev } \\
\%\end{array}$} & \multicolumn{2}{|l|}{$95 \% \mathrm{Cl}$} & \multirow[t]{2}{*}{ Cases } & \multirow[t]{2}{*}{ Pop $^{a}$} & \multirow{2}{*}{$\begin{array}{l}\text { Prev } \\
\%\end{array}$} & \multicolumn{2}{|c|}{$95 \% \mathrm{Cl}$} & \multirow[t]{2}{*}{ Cases } & \multirow[t]{2}{*}{ Pop $^{a}$} & \multirow{2}{*}{$\begin{array}{l}\text { Prev } \\
\%\end{array}$} & \multicolumn{2}{|l|}{$95 \% \mathrm{Cl}$} & \\
\hline & & & & Lower & Upper & & & & Lower & Upper & & & & Lower & Upper & \\
\hline $0-18$ & 1604 & 490,339 & 0.33 & 0.31 & 0.34 & 1163 & 461,531 & 0.25 & 0.24 & 0.27 & 2767 & 951,870 & 0.29 & 0.28 & 1.30 & 1.30 \\
\hline $19-44$ & 2791 & 851,677 & 0.33 & 0.32 & 0.34 & 2487 & 866,373 & 0.29 & 0.28 & 0.30 & 5278 & $1,718,050$ & 0.31 & 0.30 & 1.14 & 1.14 \\
\hline $45-64$ & 10,511 & 826,673 & 1.27 & 1.25 & 1.30 & 7346 & 900,610 & 0.82 & 0.80 & 0.83 & 17,857 & $1,727,283$ & 1.03 & 1.02 & 1.56 & 1.56 \\
\hline $65-74$ & 13,302 & 281,274 & 4.73 & 4.65 & 4.81 & 8474 & 327,045 & 2.59 & 2.54 & 2.65 & 21,776 & 608,319 & 3.58 & 3.53 & 1.83 & 1.83 \\
\hline $75-84$ & 18,746 & 189,776 & 9.88 & 9.74 & 10.01 & 14,381 & 257,535 & 5.58 & 5.50 & 5.67 & 33,127 & 447,311 & 7.41 & 7.33 & 1.77 & 1.77 \\
\hline $85+$ & 8578 & 60,502 & 14.18 & 13.90 & 14.46 & 10,074 & 123,175 & 8.18 & 8.03 & 8.33 & 18,652 & 183,677 & 10.15 & 10.02 & 1.73 & 1.73 \\
\hline Total & 55,532 & $2,700,241$ & 2.06 & 2.04 & 2.07 & 43,925 & $2,936,269$ & 1.50 & 1.48 & 1.51 & 99,457 & $5,636,510$ & 1.76 & 1.75 & 1.37 & 1.37 \\
\hline \multicolumn{2}{|c|}{ STD Rate ${ }^{b}$} & & 2.35 & 2.33 & 2.37 & & & 1.39 & 1.38 & 1.40 & & & 1.80 & 1.78 & 1.81 & 1.69 \\
\hline
\end{tabular}

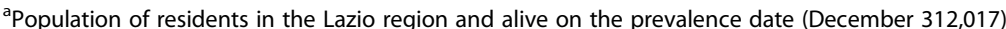

brevalence*100 standardized to the Italian ISTAT population (December 312,017)

The validity analysis on the ability of the algorithm to identify chronic dialysis patients showed sensitivity of $99.8 \%$, specificity $99.9 \%$, positive predictive value of $81.8 \%$ and negative predictive value $100.0 \%$.

\section{Discussion}

Our study represents the first experience in evaluating CKD in a large Italian region using a complex algorithm based on administrative data. It was based on multiple data collection from several databases and identified 99, 457 individuals with CKD. The crude prevalence rate of CKD in the Lazio region was $1.76 \%$ (2.06\% for males and $1.50 \%$ for females), with an increasing trend for higher age groups (up to $10.15 \%$ for patients with more

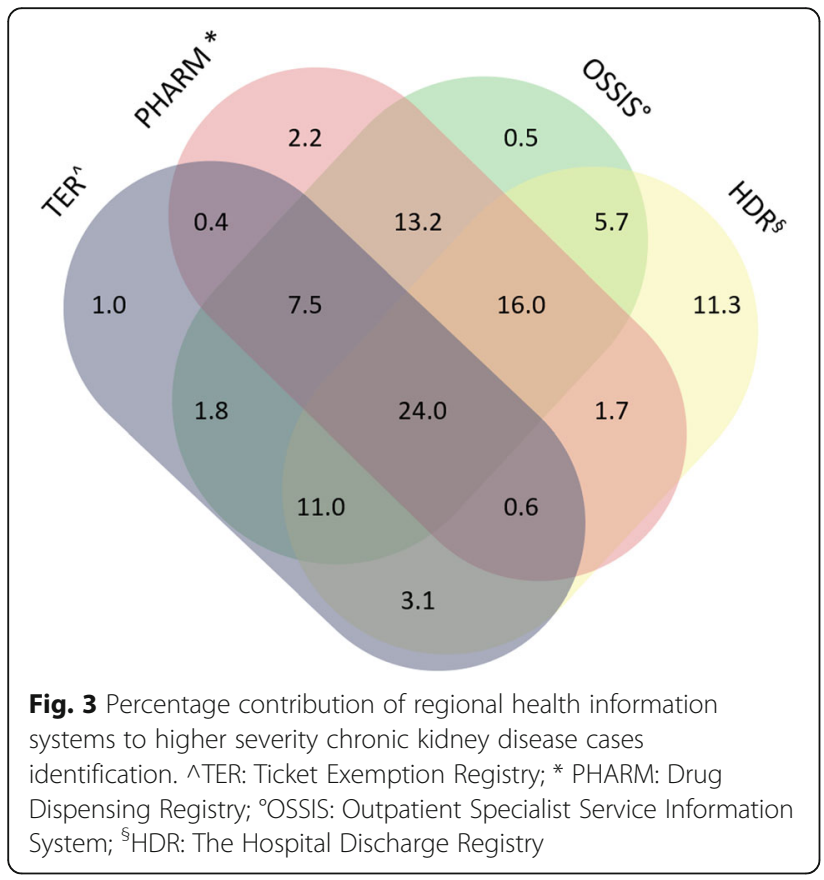

than 85 years old). Higher severity cases were $21.3 \%$ of the entire CKD population.

Estimating CKD prevalence is rather complicated. In Italy, some studies managed to predict the exact prevalence of this condition only in certain geographical areas of the country. For instance, the INCIPE study showed a prevalence of $13.2 \%$ in the Italian northeastern population older than 40 years, but its methodology is difficult to apply for the entire country and requires dedicated funding and resources [17]. Newer research models tried to improve CKD detection by including general data. In fact, using information from the 2009 and 2010 Health Survey for England, a recent model was created to estimate and predict CKD prevalence among general population, through the collection of data regarding serum creatinine (response dichotomous variable: estimated glomerular filtration rate $(\mathrm{eGFR})>$ or $<60 \mathrm{~mL} / \mathrm{min}$ per $1.73 \mathrm{~m}^{2}$ ), demographic variables (age, sex, ethnicity), area level variables (tenure, vehicle ownership, general health) and limiting long term illness. Therefore, serum creatinine was also needed in order to apply this multilevel small area synthetic estimation (ML-SASE) methodology [18].

To overcome this limitation, administrative data was considered for CKD prevalence analysis. This method is actually used by the US Renal Data System for CKD surveillance. This system defines patients with CKD through the presence of at least 1 ICD-9-CM diagnosis code from inpatient claims or at least 2 from outpatient claims or physician and supplier service claims for kidney disease [19].

As already mentioned, the successful use of administrative data for epidemiological purposes was fully achieved for diabetes, cardiovascular disease, and other diseases with relevant social and public heath impact [20-22] but it is more complex for CKD. In fact, a systematic review, including 25 observational studies from 13 administrative databases, used validated renal diagnostic codes and procedures for AKI and CKD to 


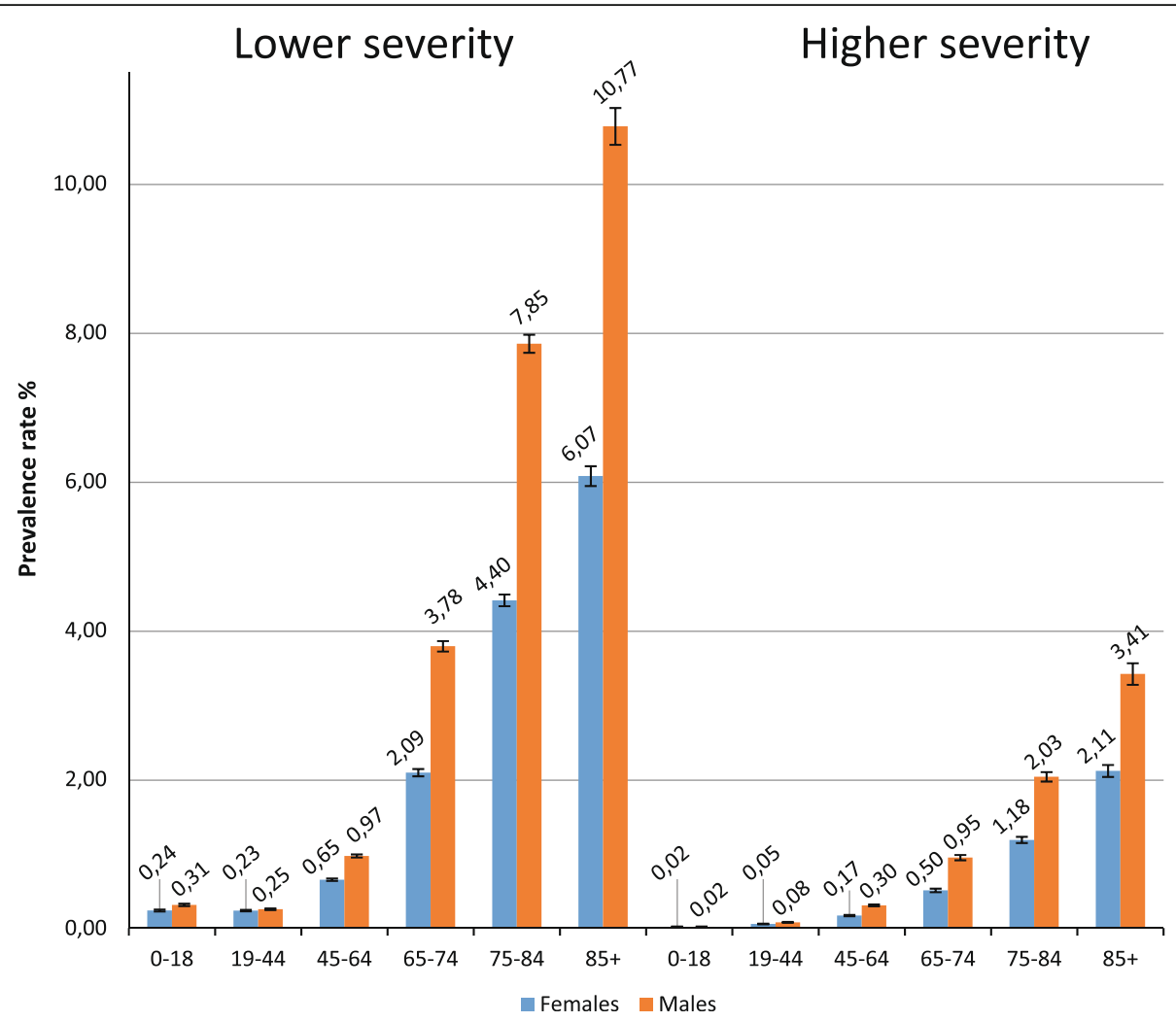

Fig. 4 Chronic kidney disease prevalence rates per 100 population by age, gender and severity, Lazio region, Italy

evaluate kidney disease against the reference laboratory standard [13]. Authors found low sensitivity and variable positive predictive value regarding CKD alone (41 and $78 \%$ respectively), albeit a better result was shown for dialysis only. To confirm these relatively poor results, Ronksley et al. examined the efficacy of an algorithm derived from administrative data to establish the epidemiology of CKD in Alberta, compared to eGFR. They used data from physician billing and hospital discharge abstracts, resulting in low sensitivity and positive predictive value when compared to clinical data (sensitivity of $19.4 \%$, specificity of $97.2 \%$, Positive Predictive Value of $60.1 \%$ and Negative Predictive Value of $84.8 \%$ ) [23].

Health administrative databases have been widely used in Italy, as in many other countries, in the last decades for epidemiological purposes. The large amount of clinical information included in these datasets allow identification of acute and chronic health conditions. Disease-specific caseidentification algorithms that combine information coded in multiple databases at a population level have been developed for many clinical conditions in the last years [24]. The systematic approach, the coverage at population level, the standardized methodology of data collection, and the large numbers are the main advantages of health information systems in order to describe the epidemiological burden of diseases and monitor the quality of a care.
However, the use of these datasets have some limitations. Completeness and accurateness of data collected may be not adequate leading to potential misclassification of the case and its severity. Moreover, the heterogeneity in the quality of coding across different institutions, for example between private and public hospitals, may lead to difficulty in interpreting the variability in the occurrence of the disease. In our study, to identify CKD cases we used hospital discharge diagnoses, prescriptions of medications, disease-specific ticket exemption and drug prescriptions that all have a good level of good quality and completeness [20-22].

To compare our main results with the existing Italian literature about CKD epidemiology, we analyzed data from three major studies where CKD was defined, according to KDIGO definition, by evaluating eGFR and albumin excretion rate. The INCIPE study evidenced an age- and gender-adjusted CKD prevalence among the general population older than 40 years of northeastern Italy of $13.2 \%$ [17]. The GUBBIO study (central Italy) was conducted on 4574 subjects in the age range 18-95 years and highlighted a prevalence of CKD stage 3-5 of $5.7 \%$ for men and $6.2 \%$ for women [25]. These studies included only regional population of Italy and since CKD is a multifactorial heterogeneous condition, it is not possible to match their results with other regional 
datasets. On the contrary, the CARHES study analyzed a better geographically distributed Italian population composed of 7552 individuals aged between 35 and 79 years, resulting in a prevalence of $7.05 \%$ (7.54\% for men and $6.54 \%$ for women). More specifically, the CKD prevalence of central Italy region was lower $(5.60 \%)$ [4].

In our case, using administrative data, we estimated in Lazio, a central region of Italy, a CKD prevalence for age $45-84$ of $2.62 \%$. Although CKD in central regions of Italy seems to be less common, these numbers are still lower than expected based on the available epidemiology data [4]. On the other hand, in $\geq 85$ years old people, CKD was much more common (prevalence of $10.15 \%$ ), consistent with published data [2]. A possible explanation for these results is related to the data used by the algorithm, since they were available only for patients who resorted to at least one of the regional health systems in a 5 years period, reflecting more severe stages of CKD that actual led to access to care. In fact, it is less probable for patients with CKD stages 1-2 to attend medical services due to their CKD condition compared with CKD stages $3-5$ subjects. This interpretation is supported by the similar prevalence of CKD stages 3-5 in the CARHES population aged 35-79 years and in our population aged $45-84: 2.89 \%$ vs $2.62 \%$, respectively. The same study showed a slightly higher CKD stage 3-5 prevalence in female subjects (males $2.76 \%$, females $3.03 \%)$. On the contrary, our results showed increased rates of CKD in males (males $3.28 \%$, females $2.04 \%$ ). According to a recent review that evaluates Italian experience in using administrative data to describe CKD epidemiology, there are very few studies on this topic [14]. In Canada, Tonelli et al. on behalf of the Alberta Kidney Disease Network used an algorithm based on hospitalization and ambulatory sensitive care condition system and found a prevalence of CKD in the population aged $18+$ of $2.9 \%$. When they added the estimated glomerular filtration rate and albuminuria, they found a prevalence of $20.4 \%$ [26, 27]. In the same ages we found a prevalence of $2.1 \%$ The heterogeneous methodology does not allow easy comparison of results [13, 14, 24]. In our study, we also tried to stratify CKD patients into groups characterized by higher and lower severity. Higher severity patients, defined by a combination of diagnostic codes of more advanced stages of CKD, need for renal replacement treatments and the prescription of drugs usually reserved for more advanced stages of disease, were found to represent slightly more than $20 \%$ of the whole population. Noteworthy, the fact that about $80 \%$ of the CKD population was defined as lower severity would suggest that our algorithm could effectively recognize less advanced stages of CKD where early interventions would be potentially more efficacious. Although we could not directly validate the accuracy of our stratification, it is interesting to note that the proportion of patients accessing all the main health systems explored increased from $5.1 \%$ (Fig. 2) in the overall CKD population to $24.0 \%$ (Fig. 3) in the higher severity group, an indirect proof of more severe disease demanding more extensive access to healthcare. In a previous study, the authors attempted to stratify patients into stages of CKD severity based on information obtained from informative systems such as ICD-10 codes, need for dialysis and prescription of drugs [28]. The authors found that such criteria were not able to discriminate between patients with eGFR below or above $90 \mathrm{~mL} / \mathrm{min} / 1.73 \mathrm{~m}^{2}$, however the specificity and negative predictive value at a threshold of $60 \mathrm{~mL} / \mathrm{min} / 1.73 \mathrm{~m}^{2}$ were very good. It is conceivable that the criteria used in our study to differentiate between patients with higher and lower severity might have similar properties.

The strengths of this study are the population-level approach and the use of standardized methodology for record-linkage procedures across multiple sources [2022]. Since most regions in Italy use the same infrastructure for health data collection, our algorithm could be applied and validated in other regional contests. Our algorithm is strengthened by the poor selection bias (all the citizens in Italy have access to healthcare independent of census and the informative resources are generated automatically, thus ensuring complete coverage of the area), reduced misclassification error regarding the chronicity of kidney, high specificity as proven by the link with the dialysis registry and a large follow-up time. The major limitation is the lack of a gold standard for the determination of renal function. Previous studies have validated their algorithm with GFR estimating equations based on serum creatinine [28]. However, eGFR alone (e.g. without information on markers of kidney damage such as proteinuria or hematuria) is a potential source of overestimation of CKD, especially in the elder population [28]. Studies based on health information systems that use a real gold-standard for renal function such as iohexol clearance are not feasible.

\section{Conclusions}

In conclusion, our new integrated algorithm based on data from different Health Information Systems in the Lazio region seems to be a useful tool for estimating the prevalence of subjects with CKD. The regional government can use it to guide the development and implementation of evidence-based pathways of care for CKD patients. Health care professionals should consider carefully the higher prevalence of lower severity CKD, since referral to specialists and optimal management of early stages may reduce the risk of worsening symptoms and complications. 


\section{Abbreviations}

95\%Cl: 95\% Confidence Intervals; ATC: Anatomical Therapeutic Chemical Classification System; CKD: Chronic kidney disease; ESRD: End-stage renal disease; HDR: The Hospital Discharge Registry; ICD-9-CM: International Classification of Disease-Ninth Revision-Clinical Modification; OSSIS: Outpatient Specialist Service Information System; PHARM: Drug Dispensing Registry; SD: Standard deviation; TER: Ticket Exemption Registry

\section{Acknowledgments}

We are grateful to the members of Dialysis and Transplant Lazio Region Registry Scientific Committee (Carmine De Cicco, Salvatore Di Giulio, Anteo Di Napoli, Sandro Feriozzi, Giuseppe Grandaliano, Isabella Guzzo, Paolo Menè, Santo Morabito, Nicola Petrosillo, Luigi Tazza, Giuseppe Tisone, Nicola Torlone) for their precious comments.

\section{Authors' contributions}

CM: Conception, design, analysis and interpretation of data, drafting the article. PMF: Conception, design, revising the article. MB: Provided intellectual content of critical importance to the work described and drafting the article. SC: Support the analytical approach, interpretation of data, revising the article. NA: Conception, design, Interpretation of data, revising the article. GG: Provided intellectual content of critical importance to the work described and reviewed the article. MD: Interpretation of data, revising the article. All authors read and approved the final manuscript and accepted personal responsibility for their contributions, and ensured the accuracy and integrity of all parts of the work, even those in which they were not personally involved.

\section{Funding}

This research did not receive any specific grant from funding agencies in the public, commercial, or not-for-profit sectors.

\section{Availability of data and materials}

Data related to the findings reported in our manuscript are available to all interested researchers upon reasonable request and with the permission of the Regional Department, because of stringent legal restrictions regarding the privacy policy on personal information in Italy (national legislative decree on privacy policy n. 196/30 June 2003). For these reasons our dataset cannot be made available on public data deposition.

\section{Ethics approval and consent to participate}

Ethics committee or institutional review board approval and informed consent were not necessary because the authors used the data already collected at the beginning of the study, and the data were analyzed anonymously through a standardized methodology according to the Italian national privacy law (national legislative decree on privacy policy no. 196/30 June 2003). The Department of Epidemiology of the Lazio Regional Health Service is the regional referral center for epidemiological research and it has full access to anonymized health information systems. Data are routinely collected by the health information system from the Regional Department of the Lazio Regional Health Service, which anonymized all the records prior to the analysis performed by our Department of Epidemiology of the Lazio Regional Health Service. The authors did not anonymize the data. The linkage method used a unique anonymous patient identifier deriving from information on persons' names, date and place of birth, and gender, according to Italian privacy legislation. Individuals cannot be identified directly or through identifiers, and the results are shown in aggregate form.

\section{Consent for publication}

Not applicable.

\section{Competing interests}

The authors declare that they have no competing interests.

\section{Author details}

'Department of Epidemiology Lazio Regional Health Service, Via Cristoforo Colombo, 112, 00147 Roma, Italy. ${ }^{2}$ U.O.C. Nefrologia, Fondazione Policlinico Universitario A. Gemelli IRCCS, Largo Agostino Gemelli, 8, 00168 Roma, Italy. ${ }^{3}$ Università Cattolica del Sacro Cuore, Roma, Largo Francesco Vito, 1, 00168 Roma, Italy. ${ }^{4}$ Department of Medicine, Renal Unit, Division of Nephrology and Dialysis, University of Verona, Piazzale Ludovico Antonio Scuro 10, 37134 Verona, Italy.

Received: 4 September 2019 Accepted: 14 January 2020

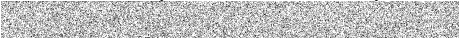

\section{References}

1. Webster AC, Nagler EV, Morton RL, Masson P. Chronic kidney disease. Lancet. 2017:389:1238-52. https://doi.org/10.1016/S0140-6736(16)32064-5.

2. Hill NR, Fatoba ST, Oke JL, et al. Global prevalence of chronic kidney disease - a systematic review and meta-analysis. PLoS One. 2016;11:e0158765. https://doi.org/10.1371/journal.pone.0158765.

3. Global Burden of Disease Study. Collaborators (2015) global, regional, and national incidence, prevalence, and years lived with disability for 301 acute and chronic diseases and injuries in 188 countries, 1990-2013: a systematic analysis for the global burden of disease study 2013. Lancet. 2013;386:743800. https://doi.org/10.1016/S0140-6736(15)60692-4.

4. De Nicola L, Donfrancesco C, Minutolo R, et al. Prevalence and cardiovascular risk profile of chronic kidney disease in Italy: results of the 2008-12 National Health Examination Survey. Nephrol Dial Transplant. 2015; 30:806-14. https://doi.org/10.1093/ndt/gfu383.

5. Wyld MLR, Lee CMY, Zhuo X, et al. Cost to government and society of chronic kidney disease stage 1-5: a national cohort study. Intern Med J. 2015:45:741-7. https://doi.org/10.1111/imj.12797.

6. Jommi C, Armeni P, Battista M, et al. The cost of patients with chronic kidney failure before Dialysis: results from the IRIDE observational study. Pharmacoecon Open. 2018;2:459-67. https://doi.org/10.1007/s41669-017-0062-z.

7. Trivedi $\mathrm{H}$. Cost implications of caring for chronic kidney disease: are interventions cost-effective? Adv Chronic Kidney Dis. 2010;17:265-70. https://doi.org/10.1053/j.ackd.2010.03.007.

8. Turchetti G, Bellelli S, Amato M, et al. The social cost of chronic kidney disease in Italy. Eur J Health Econ. 2017;18:847-58. https://doi.org/10.1007/ s10198-016-0830-1.

9. Cicchetti A, Ruggeri M, Codella P. Ridolfi A. I costi socio-sanitar dell'insufficienza renale cronica. 2011:12:75-82

10. Berloco P (2016) Documento di indirizzo per la malattia renale cronica. http://www.salute.gov.it/portale/documentazione/p6_2_2_1.jsp?id=2244. Accessed 24 Jun 2019

11. Hux JE, Ivis F, Flintoft V, Bica A. Diabetes in Ontario: determination of prevalence and incidence using a validated administrative data algorithm Diabetes Care. 2002;25:512-6. https://doi.org/10.2337/diacare.25.3.512.

12. Quan $\mathrm{H}$, Khan $\mathrm{N}$, Hemmelgarn $B R$, et al. Validation of a case definition to define hypertension using administrative data. Hypertension. 2009;54:14238. https://doi.org/10.1161/HYPERTENSIONAHA.109.139279.

13. Vlasschaert MEO, Bejaimal SAD, Hackam DG, et al. Validity of administrative database coding for kidney disease: a systematic review. Am J Kidney Dis. 2011;57:29-43. https://doi.org/10.1053/j.ajkd.2010.08.031.

14. Di Domenicantonio R, Cappai G, Agabiti N, et al. A systematic review of case-identification algorithms based on Italian healthcare administrative databases for three relevant diseases of the digestive and genitourinary system: inflammatory bowel diseases, celiac disease, and chronic kidney disease. E\&P. 2019;43:88-98. https://doi.org/10.19191/EP19.4.S2.P088.095.

15. Di Domenicantonio R, Cappai G, Cascini S, et al. Validation of algorithms for the identification of subjects with chronic disease using health information systems. Epidemiol Prev. 2018:42:316-25. https://doi.org/10.19191/EP18.5-6. P316.100.

16. Khan KS, Chien PF. Evaluation of a clinical test. I: assessment of reliability. BJOG. 2001;108:562-7.

17. Gambaro G, Yabarek T, Graziani MS, et al. Prevalence of CKD in northeastern Italy: results of the INCIPE study and comparison with NHANES. Clin J Am Soc Nephrol. 2010;5:1946-53. https://doi.org/10.2215/CJN.02400310.

18. Aitken GR, Roderick PJ, Fraser S, et al. Change in prevalence of chronic kidney disease in England over time: comparison of nationally representative cross-sectional surveys from 2003 to 2010. BMJ Open. 2014;4: e005480. https://doi.org/10.1136/bmjopen-2014-005480.

19. Collins AJ, Chen S-C, Gilbertson DT, Foley RN. CKD surveillance using administrative data: impact on the health care system. Am J Kidney Dis 2009:53:S27-36. https://doi.org/10.1053/j.ajkd.2008.07.055

20. Cascini S, Agabiti N, Incalzi RA, et al. Pneumonia burden in elderly patients: a classification algorithm using administrative data. BMC Infect Dis. 2013;13: 559. https://doi.org/10.1186/1471-2334-13-559. 
21. Di Domenicantonio R, Cappai G, Arcà M, et al. Occurrence of inflammatory bowel disease in Central Italy: a study based on health information systems. Dig Liver Dis. 2014;46:777-82. https://doi.org/10.1016/j.dld.2014.04.014.

22. Bargagli AM, Colais P, Agabiti N, et al. Prevalence of multiple sclerosis in the Lazio region, Italy: use of an algorithm based on health information systems. J Neurol. 2016;263:751-9. https://doi.org/10.1007/s00415-016-8049-8.

23. Ronksley PE, Tonelli M, Quan $\mathrm{H}$, et al. Validating a case definition for chronic kidney disease using administrative data. Nephrol Dial Transplant. 2012;27: 1826-31. https://doi.org/10.1093/ndt/gfr598.

24. Canova C, Simonato L, Barbiellini Amidei C, et al. A systematic review of case-identification algorithms for 18 conditions based on Italian healthcare administrative databases: a study protocol. Epidemiol Prev. 2019;43:8-16. https://doi.org/10.19191/EP19.4.S2.P008.089.

25. Cirillo M, Terradura-Vagnarelli O, Mancini M, et al. Cohort profile: the Gubbio population study. Int J Epidemiol. 2014;43:713-20. https://doi.org/10.1093/ ije/dyt025.

26. Tonelli M, Wiebe N, Fortin M, Alberta kidney disease network, et al. Methods for identifying 30 chronic conditions: application to administrative data. BMC Med Inform Decis Mak. 2015;17(15):31. https://doi.org/10.1186/s12911015-0155-5.

27. Ronksley PE, Tonelli M, Quan $\mathrm{H}$, et al. Alberta kidney disease network. Validating a case definition for chronic kidney disease using administrative data. Nephrol Dial Transplant. 2012;27(5):1826-31. https://doi.org/10.1093/ ndt/gfr598.

28. Friberg L, Gasparini A, Carrero JJ. A scheme based on ICD-10 diagnoses and drug prescriptions to stage chronic kidney disease severity in healthcare administrative records. Clin Kidney J. 2018;11:254-8. https://doi.org/10.1093/ ckj/sfx085.

\section{Publisher's Note}

Springer Nature remains neutral with regard to jurisdictional claims in published maps and institutional affiliations.

Ready to submit your research? Choose BMC and benefit from:

- fast, convenient online submission

- thorough peer review by experienced researchers in your field

- rapid publication on acceptance

- support for research data, including large and complex data types

- gold Open Access which fosters wider collaboration and increased citations

- maximum visibility for your research: over $100 \mathrm{M}$ website views per year

At $\mathrm{BMC}$, research is always in progress.

Learn more biomedcentral.com/submissions 